\title{
Are Platelets Count Useful for Detecting the Grade of Steatosis?
}

\author{
Kento Imajo ${ }^{1}$; Masato Yoneda ${ }^{1,2}$; Atsushi Nakajima ${ }^{1, *}$ \\ ${ }^{1}$ Department of Gastroenterology and Hepatology, Yokohama City University Graduate School of Medicine, Yokohama, Japan \\ ${ }^{2}$ Schiff Center for Liver Diseases, University of Miami Miller School of Medicine, Miami, USA \\ *Corresponding Author: Atsushi Nakajima, Department of Gastroenterology and Hepatology, Yokohama City University Graduate School of Medicine, Yokohama, Japan. Tel: +81- \\ 457872640, Fax: +81-457843546, E-mail: nakajima-tky@umin.ac.jp \\ Received: March 31, 2015; Accepted: April 8, 2015
}

Keywords: Blood Platelets; Fibrosis; Fatty Liver

\section{Dear Editor,}

We read the article by Garjani et al. (1) with great interest. In this study, they demonstrated that patients with moderate or severe fatty liver disease on ultrasonography had higher platelet counts than those with mild fatty liver and concluded that platelet counts could be used as a clue to steatosis grade of nonalcoholic fatty liver disease (NAFLD). Low platelet counts are frequently observed in patients with several liver diseases (2) and measurement of the platelet count is clinically important for prediction of liver fibrosis in patients with chronic liver disease. However, studies investigating the association between steatosis and platelet count are rare. Therefore, Garjani's indication that platelet count was elevated by increase in ultrasonographic grade of steatosis is a very unique viewpoint. In this study, they stated some limitations. The most important limitation was that no histological information of the liver was available in their study. Recently, we reported the association between platelet counts and histologic fibrosis severity of NAFLD using the Japanese Society Group (JSG)-NAFLD database (3). In this study, 1048 patients with biopsy-proven NAFLD were enrolled and considered to be one of the largest cohorts in the world (3). We re-analyzed the association between platelet counts and histological grade of steatosis using this database like Garjani et al. (1). The results showed that platelet counts were lower in patients with mild steatosis (Steatosis grade 1) compared to moderate or severe steatosis (steatosis grades 2-3) (21.9 \pm 7.2 in grade 1, $23.1 \pm 5.9$ in grade 2, $23.5 \pm 6.0$ in grade $3, \mathrm{P}=0.0021$ by Kruskal-Wallis test) as Garjani reported. However, when we excluded patients with severe fibrosis (fibrosis stages 3-4), the platelet counts did not correlate with steatosis grade in our cases $(23.5 \pm 6.8$ in grade 1, 23.6 \pm 5.6 in grade 2, $24.2 \pm$ 5.9 in grade $3, P=0.2029$ by Kruskal-Wallis test). Decrease in steatosis grade was well known to be observed as progression to advanced liver fibrosis, especially cirrhosis in patients with NAFLD (4). Therefore, it is necessary to evaluate the status of fibrosis to investigate whether steatosis increases platelet count. Even if there are no patients who had an enlarged spleen on ultrasonography in their study, elastography (5) and some scoring systems that do not involve platelet count should be used to exclude NAFLD cases with advanced fibrosis. Since the incidence of NAFLD is rising rapidly in both adults and children, the biomarker is of major clinical interest. Platelet count has historically been measured to assess liver fibrosis by many clinicians, because it is simple, easy to measure and cost-effective. Additional information from other institutions from many countries may also be needed to clarify the association between platelet count and steatosis. We hope this manuscript be useful to further investigate the role of platelet count in patients with NAFLD.

\section{Acknowledgements}

Work in the authors' laboratory was supported by a program from the Japan Science and Technology Agency (J.S.T.) and Kiban-B, Shingakujuturyouiki. In addition, the present work was supported in part by grants-in-aid from the Japanese Ministry of Health, Labour and Welfare.

\section{Authors' Contributions}

Kento Imajo wrote the manuscript. Masato Yoneda and Atsushi Nakajima revised the manuscript.

\section{References}

1. Garjani A, Safaeiyan A, Khoshbaten M. Association between platelet count as a noninvasive marker and ultrasonographic grading in patients with nonalcoholic Fatty liver disease. Hepat Mon. 2015;15(1): e24449.

2. Afdhal N, McHutchison J, Brown R, Jacobson I, Manns M, Poordad $\mathrm{F}$, et al. Thrombocytopenia associated with chronic liver disease. J Hepatol. 2008;48(6):1000-7.

3. Yoneda M, Fujii H, Sumida Y, Hyogo H, Itoh Y, Ono M, et al. Platelet count for predicting fibrosis in nonalcoholic fatty liver disease. $J$

Copyright ( 2015, Kowsar Corp. This is an open-access article distributed under the terms of the Creative Commons Attribution-NonCommercial 4.0 International License (http://creativecommons.org/licenses/by-nc/4.0/) which permits copy and redistribute the material just in noncommercial usages, provided the original work is properly cited. 
Gastroenterol. 2011:46(11):1300-6.

4. Neuschwander-Tetri BA, Caldwell SH. Nonalcoholic steatohepatitis: summary of an AASLD Single Topic Conference. Hepatology. 2003;37(5):1202-19.
5. Yoneda M, Yoneda M, Mawatari H, Fujita K, Endo $\mathrm{H}$, Iida $\mathrm{H}$, et al. Noninvasive assessment of liver fibrosis by measurement of stiffness in patients with nonalcoholic fatty liver disease (NAFLD). Dig Liver Dis. 2008;40(5):371-8. 